\title{
Malignant Pulmonary Artery Neoplasm
}

National Cancer Institute

\section{Source}

National Cancer Institute. Malignant Pulmonary Artery Neoplasm. NCI Thesaurus. Code C5380.

A malignant neoplasm involving the pulmonary artery. 